\title{
Enhanced Energetic Neutral Atom Imaging
}

\author{
Earl E. Scime ${ }^{1 *}$ and Amy M. Keesee ${ }^{2}$ \\ ${ }^{1}$ Department of Physics and Astronomy, West Virginia University, Morgantown, WV, United States, ${ }^{2}$ Department of Physics \\ and Space Science Center, University of New Hampshire, Durham, NH, United States
}

Over the past two decades, instruments designed to image plasmas in energetic neutral atom (ENA) emission have flown in space. In contrast to typical satellite-based in situ instruments, ENA imagers provide a global view of the magnetosphere because they remotely measure ion distributions via neutrals that are not tied to the magnetic field. An intrinsic challenge that arises during analysis of magnetospheric ENA images is that the ENA fluxes are integrated along the line-of-sight of the instrument. We propose a method of enhancing ENA emission from a localized region in space, thereby enabling spatially resolved measurements of ENA emission in a remotely obtained ENA image. Here we

OPEN ACCESS

Edited by:

Gian Luca Delzanno,

Los Alamos National Laboratory

(DOE), United States

Reviewed by:

Mike Gruntman,

University of Southern California,

United States

Herbert Funsten,

Los Alamos National Laboratory

(DOE), United States

*Correspondence:

Earl E. Scime

escime@wvu.edu

Specialty section:

This article was submitted to

Space Physics,

a section of the journal

Frontiers in Astronomy and Space

Sciences

Received: 27 September 2018

Accepted: 30 January 2019

Published: 20 February 2019

Citation:

Scime EE and Keesee AM (2019)

Enhanced Energetic Neutral Atom

Imaging.

Front. Astron. Space Sci. 6:9.

doi: 10.3389/fspas.2019.00009 show that releases of modest volumes $\left(\sim 1.4 \mathrm{~m}^{3}\right)$ of liquid hydrogen in space are sufficient to accomplish the ENA localization.

Keywords: magnetosphere, active space experiment, energetic neutral atoms, charge exchange, geocorona, ion energy spectra

\section{INTRODUCTION}

In the late 1980's, Roelof realized that energetic particle signals obtained during cusp transits by the IMP 7/8 and ISEE-1 spacecraft could be explained if the signals were actually energetic neutral atoms that had escaped the inner magnetosphere after charge exchange collisions with energetic ions (Roelof et al., 1985; Roelof, 1987). After he demonstrated that the measurements could be used to create neutral atom images of the inner magnetosphere, the space plasma community embraced the concept of energetic neutral atom imaging. A remote sensing technique, energetic neutral atom (ENA) imaging provides global views of magnetospheric ion populations that have been processed through charge exchange collisions with the Earth's cold geocorona (Scime and Zaniewski, 2004). Where the geocorona coexists with hot plasma, fast ions undergo charge exchange collisions with geocoronal neutral atoms; producing a cold ion and an energetic neutral atom with the same energy as the original ion. By its very nature, the ENA production process yields a flux of ENAs along every line-of-sight through the magnetosphere. Since the cross section for charge exchange collisions between ions and neutrals is well known from laboratory measurements, with a model of the shape and density of the geocorona it is possible to convert energy-resolved ENA measurements into a measure of the line-integrated ion energy spectrum along a given line-of-sight.

For readers interested in an in-depth discussion of neutral atom imaging, Gruntman's review of the history of neutral atom imaging is an excellent resource (Gruntman, 1997). The first ENA imager of the "modern" era, the Ion Neutral Camera (INCA), successfully obtained ENA images of the Saturnian magnetosphere from aboard the Cassini spacecraft (Mitchell et al., 2000). The instrument complement of the Imager for Magnetopause-to-Aurora Global Exploration (IMAGE) 
spacecraft included three different ENA cameras. The Low [LENA; (Moore et al., 2000)], Medium [MENA; (Pollock et al., 2000)], and High [HENA; (Mitchell et al., 2000)] energy neutral atom imagers. The energy range of those instruments spanned $15 \mathrm{eV}$ to $500 \mathrm{keV}$ per nucleon. Using the same medium energy instrument design as IMAGE, the two ENA imagers of the TWINS mission (McComas et al., 2009a) have provided nearly a decade of continuous ENA observations of the terrestrial magnetosphere (Keesee and Scime, 2015). Using a different technique to image medium energy neutrals created at the heliospheric termination shock, the IBEX-HI ENA imager aboard the Interstellar Boundary Explorer (IBEX) spacecraft has revolutionized our understanding of the structure of the outer heliosphere (McComas et al., 2009b).

ENA imaging of the magnetosphere has also provided a wealth of information about ion dynamics during geomagnetic storms and substorms. Roelof found a strong day-night asymmetry in the ion ring current during a geomagnetic storm using ISEE 1 ENA data (Roelof, 1987). Pollock used MENA data to demonstrate the evolution from partial to complete ring current during a storm as well as that the loss of ring current ions is dominantly through the dayside magnetopause (Pollock et al., 2001). C:son Brandt used HENA data to show the existence of strong, skewed equatorial electric fields in the inner magnetosphere that depend upon the solar wind velocity and interplanetary magnetic field (IMF) $\mathrm{B}_{y}$ (C:son Brandt et al., 2002b). Keesee et al. (2014) used TWINS images to show the propagation of regions of energized ions from the magnetotail toward the inner magnetosphere, with downward deflection near geosynchronous orbit. Perez et al. (2016) discovered the existence of two ion flux peaks in the inner magnetosphere trapped population during a storm using TWINS data. ENA data have also been used in conjunction with modeling to study the inner magnetosphere electric field (Buzulukova et al., 2010; Fok et al., 2010), the neutral geocorona (Ilie et al., 2013a), ion composition (Ilie et al., 2013b), and the influence of boundary conditions on simulation results (Elfritz et al., 2014).

While ENA imaging has yielded important insights into the structure and evolution of the terrestrial magnetosphere, the lack of spatial resolution has held back widespread acceptance of the ion energy distribution results from ENA energy spectra measurements-even though comparative studies between ENA energy spectra and local ion energy spectra have demonstrated impressive consistency between the two techniques (Keesee et al., 2012, 2014; Perez et al., 2016; Goldstein et al., 2017). Global ENA measurements have also proved useful for model validation (e.g., Fok et al., 2014). Spatially resolved ENA measurements would provide an additional method of using ENA measurements to validate magnetospheric models while increasing the intrinsic value of ENA data.

Therefore, in the spirit of the 2017 workshop on Active Experiments in Space held in Santa Fe, NM, here we describe an active space experiment that would provide spatially resolved ENA measurements of ion energy distributions and enable researchers to distinguish signals from a specific spatial location from within the line-integrated measurement.

\section{ACTIVE ENA MEASUREMENTS}

An ENA is created through a charge exchange collision between an energetic ion and a cold neutral atom. The measured ENA intensity, $j_{\mathrm{ENA}}$ (with units of $\left.\left(\mathrm{cm}^{2} \mathrm{sr} \mathrm{s} \mathrm{eV}\right)^{-1}\right)$, depends upon the ion intensity, $j_{\text {ion }}$, the charge exchange cross section, $\sigma_{\mathrm{cx}}$ (Freeman and Jones, 1974), and the neutral density, $n_{\mathrm{n}}$, through the relation

$$
\begin{aligned}
\mathbf{j}_{\mathrm{ENA}}(\mathrm{E}, \overrightarrow{\mathbf{u}})= & \sigma_{\mathrm{cx}}(\mathrm{E}) \int_{\mathbf{0}}^{\overrightarrow{\mathbf{R}}}\left(\mathbf{n}_{\mathbf{n}}(\overrightarrow{\mathbf{r}}(\mathbf{s})) \mathbf{j}_{\mathrm{ion}}(\overrightarrow{\mathbf{r}}(\mathbf{s}), \mathrm{E}, \overrightarrow{\mathbf{u}})\right. \\
& \left.\exp \left(-\int_{\mathbf{0}}^{\overrightarrow{\mathbf{r}}(\mathbf{s})}\left(\alpha\left(\mathbf{s}^{\prime}\right) \mathbf{d} \mathbf{s}^{\prime}\right)\right) \mathbf{d} \mathbf{s}\right)
\end{aligned}
$$

where the integral is performed along the line of sight (LOS). Attenuation of ENAs due to additional collisions and photoionization is accounted for in the integral over $\alpha\left(s^{\prime}\right)$. We assume this integral is approximately zero, which is applicable to most of the magnetosphere beyond a few Earth radii.

If we assume a Maxwellian parent ion distribution, then the hottest region along the LOS dominates the high energy portion of the spectrum (Hutchinson, 1987). Under this assumption, equation (1) becomes

$$
\frac{\mathbf{j}_{\mathrm{ENA}}(\mathbf{E}, \overrightarrow{\mathbf{u}})}{\sigma_{\mathrm{cx}}(\mathbf{E}) \mathbf{E}} \approx \frac{\xi \mathbf{n}_{\mathbf{n}}\left(\mathbf{r}^{*}\right) \mathbf{n}_{\mathbf{i}}\left(\mathbf{r}^{*}\right)}{\sqrt{2 \mathbf{m}_{\mathbf{i}}}\left(\pi \mathbf{T}_{\mathbf{i}}\left(\mathbf{r}^{*}\right)\right)^{3 / 2}} \exp \left(\frac{-\mathbf{E}}{\mathbf{T}_{\mathbf{i}}\left(\mathbf{r}^{*}\right)}\right)
$$

where the integral has been approximated by the value at the hottest point, $r^{*}$, times a characteristic length along $r, \xi$ (Scime and Hokin, 1992). The Maxwellian assumption is valid for the plasma sheet but less so for the inner magnetosphere. The Maxwellian assumption method calculates an average energy of the bulk population that provides information about the energization of the ions within the measured energy range (typically 1-100 keV). The ion temperatures, $T_{i}$, are calculated by fitting Equation (2) to the measured ENA energy spectrum, a method that has been verified through comparison to in situ measurements (Scime et al., 2002).

The key term in the measured ENA flux for the active method proposed here is the density of the background neutral population, $n_{n}(\vec{r}(s))$. The geocorona surrounding the Earth is a roughly spherical distribution of cold neutral gas. Early measurements by Rairden et al. (1986) estimated the neutral hydrogen density at geosynchronous orbit $\left(6 R_{\mathrm{E}}\right)$ to be 50 $\mathrm{cm}^{-3}$. Subsequent measurements by Ostgaard et al. (2003) using extreme ultraviolet (EUV) emission measurements from the IMAGE spacecraft yielded nearly identical values for the geosynchronous neutral density. More recent measurements using EUV measurements from the TWINS mission (Bailey and Gruntman, 2011) yield daytime geosynchronous neutral densities of $100 \mathrm{~cm}^{-3}$. At night, the neutral density doubles to $200 \mathrm{~cm}^{-3}$ (Bailey and Gruntman, 2011). ENA images of the magnetotail suggest that the neutral density in the anti-sunward tail of the geocorona decreases more gradually with increasing 
distance from the Earth, consistent with a substantial night/day asymmetry in the geocoronal neutral density at geosynchronous orbit (Keesee et al., 2012).

Before describing how the ENA signal might be enhanced for a particular region in space, it is illustrative to describe how ENA images are constructed from the ENA measurements. Three methods are generally used. First, ion distributions can be calculated from the ENA data using a deconvolution technique described by Perez et al. (2000). Second, a constrained linear inversion technique as described by C:son (C:son Brandt et al., 2002a), can be used to find ion distributions that result in ENA images that are matched to the measurements. These two techniques provide a measurement of the ion density and temperature, but require accurate models of the magnetic field and neutral geocorona. The third method, the method typically used in our studies because it requires few computational resources and a minimum of assumptions, enables a calculation of only the ion temperature independent of the neutral source distribution. Typically, we map the measured ENA fluxes along the LOS to the $x y$ plane (GSM coordinates), assuming that the hottest point along the LOS occurs in the central plasma sheet (Hughes, 1995) and, therefore, near the equatorial plane in the magnetotail; which also eliminates the need for a magnetic field model. The field of view (FOV) of each pixel in an ENA image is projected along the LOS to calculate the intersection of the FOV with the GSM $x y$ plane. The ENA emissivity is placed proportionally in the $0.5 \times 0.5 R_{\mathrm{E}} x y$ plane bins with which the FOV intersects. This algorithm accounts for the increasing FOV with increasing distance from the Earth. A modeled magnetosphere boundary (Shue et al., 1997) is used to discard flux projected to bins that are outside the boundary. The average emissivity for each bin is calculated prior to calculating the ion temperature. This method of calculating projected ion temperatures has been validated with in situ measurements (Keesee et al., 2008) and a typical ENA ion temperature image is shown in Figure 1.

Given that geocoronal hydrogen densities range from 100 to $200 \mathrm{~cm}^{-3}$ at $6 R_{\mathrm{E}}$ and decrease for larger geocentric distances, an increase in the local neutral hydrogen density of $200 \mathrm{~cm}^{-3}$ would produce a significant enhancement of the ENA flux emitted from that region of space. An earlier study when the field of ENA imaging was just beginning (McComas et al., 1993), proposed that cold releases from conventional explosions in space could be detectable with the ENA instruments under development at the time. That study, with important national security implications, is a precursor to the analysis described here.

In terms of a line integrated measurement, the typical linesof-sight for the TWINS ENA imager (see Figure 2) pass through roughly $10 R_{\mathrm{E}}$ of the magnetosphere where the plasma and neutral densities are large enough to generate significant ENA fluxes. Assuming uniform ENA emission along the entire line of sight and a uniform geocoronal hydrogen density of $100 \mathrm{~cm}^{-3}$, conservative estimates, an increase of $200 \mathrm{~cm}^{-3}$ along $0.5 R_{\mathrm{E}}$ would yield a $10 \%$ increase in the total ENA signal from a given line of sight. A modulated increase would be easily detectable if

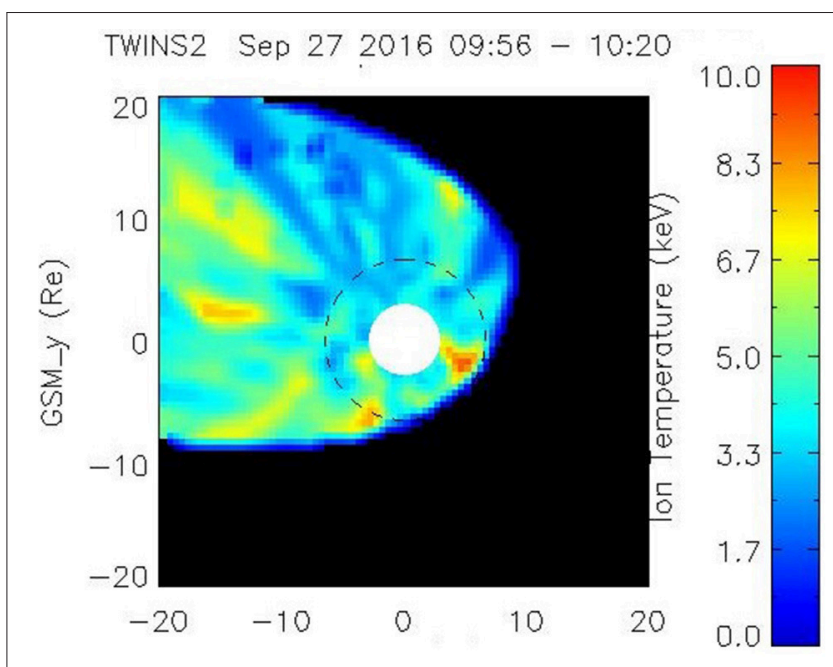

FIGURE 1 | lon temperatures in the GSM equatorial plane calculated using energetic neutral atom data from TWINS 2 on Sept. 27, 2016 at 9:56-10:20 $U T$. The white disc has radius $3 R_{\mathrm{E}}$ centered at Earth and the dashed line indicates geosynchronous orbit. The area of measured temperatures is influenced by a combination of the instrument field of view and a modeled magnetosphere boundary.

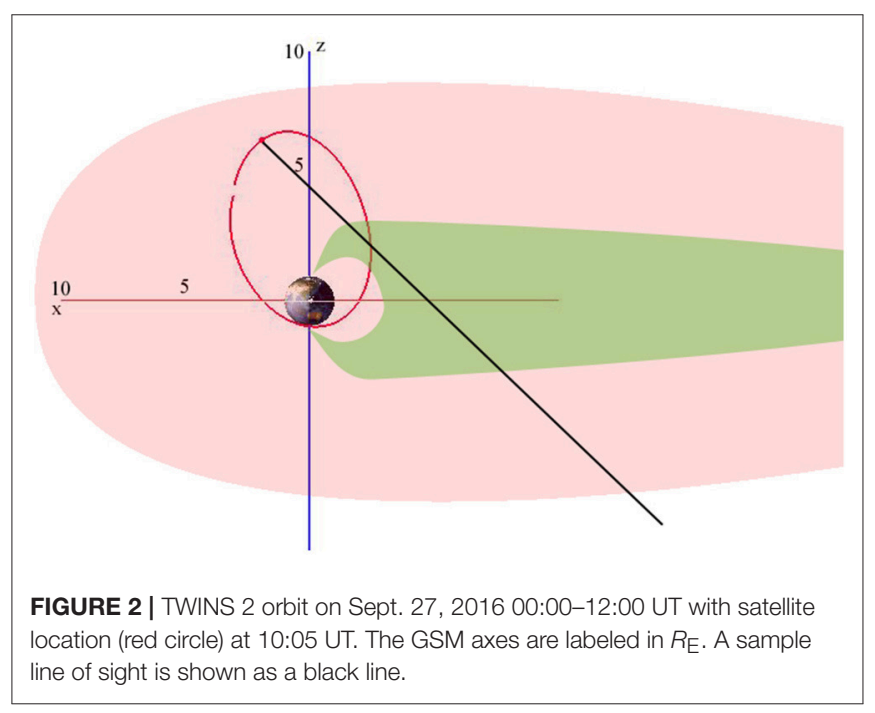

the modulation frequency was distinct from naturally occurring geophysical processes.

Based on the density of liquid hydrogen $(2.02 \mathrm{~g} / \mathrm{mol}=70.85$ $\mathrm{g} / \mathrm{l})$, an increase of $200 \mathrm{~cm}^{-3}$ in the neutral density across a spherical volume $0.5 R_{\mathrm{E}}$ in diameter requires

$$
\begin{aligned}
\text { atoms of hydrogen } & =200 \mathrm{~cm}^{-3} \times \frac{4 \pi\left(6.4 \times 10^{8} \mathrm{~cm} / 2\right)^{3}}{3} \\
\text { atoms of hydrogen } & =2.8 \times 10^{28} \\
\text { moles of hydrogen } & =46.5 \times 10^{3} . \\
\text { mass of liquid hydrogen }\left(\mathrm{H}_{2}\right) & =50 \mathrm{~kg} . \\
\text { liters of liquid hydrogen }\left(\mathrm{H}_{2}\right) & =663 \text { liters }\left(0.7 \mathrm{~m}^{-3}\right)
\end{aligned}
$$


This calculation takes advantage of the molecular nature of liquid hydrogen and therefore, to generate neutral hydrogen atoms, a dissociation process would be required, e.g., a UV light source. However, at the energies of interest, $\sim 10 \mathrm{keV}$, the charge exchange cross section for producing ENAs from collisions with molecular hydrogen, $10^{-15} \mathrm{~cm}^{2}$, is nearly identical to the cross section for neutral hydrogen (Freeman and Jones, 1974). Therefore, doubling the volume of liquid hydrogen to $1.4 \mathrm{~m}^{-3}$ would yield the required increase in the ENA emission. In other words, a cube of liquid hydrogen $1.1 \mathrm{~m}$ on a side provides enough molecular hydrogen for the desired increase in the neutral density over a spherical volume $0.5 R_{\mathrm{E}}$ in diameter.

To put this volume in perspective, the published fuel capacity of the 2nd stage of a Falcon 9 rocket is 27,634 liters of liquid oxygen and 17,413 liters of liquid kerosene (www.spaceflight101. net/falcon-9-launch-vehicle-information). One such release is therefore equivalent to $7.6 \%$ of the standard oxygen capacity by volume of the Falcon 9 2nd stage. Surreptitiously, the charge exchange cross section for protons on molecular oxygen at 10 $\mathrm{keV}$ is four times that of protons on molecular hydrogen (Allison, 1958). Therefore, an unaltered Falcon 9 2nd stage boosted into geosynchronous orbit could provide more than 40 controlled releases of liquid oxygen for an active ENA experiment of the type proposed here. There are other options for producing these ENA enhancements as well. For example, unused fuel reserves on standard commercial or scientific launches could be used to trigger the ENA emission enhancements as described here.

Neutral cloud releases are not a novel concept. Fuselier et al. (1994) optically tracked an explosive release of barium to map local magnetic field lines and to investigate the ionization processes in near-Earth space. More recently, barium releases have been proposed as a means of exciting electromagnetic ion cyclotron waves in the lower magnetosphere. Fundamentally, this proposed active experiment is much less complicated than the barium release experiments (Fuselier et al., 1994). The desired material is under pressure and release is accomplished with a standard cryogenic valve.

As the released plume expands, the spatial localization of the ENA enhancement begins. Initially, the energy spectrum of any increases in observed ENA signal can be compared to local measurements of the ion energy spectrum. As the plume expands further, the enhanced ENA emission integrates over a large volume of space and comparisons could be made to ion energy spectra in nearby regions of space. Assuming the released gas is at the boiling temperature of liquid hydrogen, $\sim 33 \mathrm{~K}$, the gas cloud will expand at $\sim 400 \mathrm{~m} / \mathrm{s}$. Comparing that escape speed to the orbital velocity of a geosynchronous spacecraft, $3,100 \mathrm{~m} / \mathrm{s}$, it is clear that for most orbital tracks, the motion of a released

\section{REFERENCES}

Allison, S. K. (1958). Experimental results on charge-changing collisions of hydrogen and helium atoms and ions at kinetic energies above $0.2 \mathrm{keV}$. Rev. Modern Phys. 30, 1137-1168. doi: 10.1103/RevModPhys.30.1137

Bailey, J., and Gruntman, M. (2011). Experimental study of exospheric hydrogen atom distributions by Lyman-alpha detectors on the TWINS mission. J. Geophys. Res. 116:A09302. doi: 10.1029/2011JA016531 cloud will be dominated by the spacecraft velocity and in that frame, a cloud would take roughly $3 \mathrm{~h}$ to expand to a radius of $1 R_{\mathrm{E}}$. An oxygen cloud would expand much more slowly even if released at the higher temperature of liquid oxygen. Because the expanding cloud will track the spacecraft velocity, the cloud will also sweep through magnetic local time in the magnetosphere as it expands-enabling the sampling of a range of magnetic local times with each release. Another factor in the lifetime of the cloud is the combined photoionization and charge exchange rate at $1 \mathrm{AU}$. For hydrogen, these two loss processes would reduce the overall cloud density by $<1 \%$ over the time it takes for the cloud to expand to $1 R_{\mathrm{E}}$ (Ogawa et al., 1995). The lifetime for an expanding oxygen cloud is similar, given the photoionization rate of oxygen at $1 \mathrm{AU}$ (Meier et al., 2007).

While the enhanced ENA emission should be localizable by a single ENA imager, the advantages of stereoscopic ENA imaging, such as that provided by the dual TWINS instruments (McComas et al., 2009a), are significant. Releases such as those proposed here would provide a unique opportunity to validate stereoscopic inversion techniques as well as identify projection issues arising from non-uniform pitch angle distributions of the parent energetic ion populations. An ideal active neutral release experiment might include multiple tanks of liquid hydrogen or oxygen placed into different regions of the magnetosphere. Individual and/or simultaneous enhancements could then be imaged with a single or multiple, full sky, ENA imagers to provide simultaneous measurements of the global ion energy spectrum from different regions of space.

\section{SUMMARY}

The next step in validating this active space ENA experimental concept would be to perform computational simulations, using existing magnetospheric models of ENA emission (Perez et al., 2001), to estimate detection thresholds and density enhancement requirements for a variety of magnetospheric conditions and viewing geometries.

\section{AUTHOR CONTRIBUTIONS}

ES conceived of the presented idea based on discussions with AK. ES performed the calculations and took the lead on the manuscript. AK contributed to the text and created the figures.

\section{FUNDING}

This work supported by NASA grant 80NSSC18K0359. 
C:son Brandt, P., Ohtani, S., Mitchell, D. G., Fok, M.-C., Roelof, E. C., and Demajistre, R. (2002b). Global ENA observations of the storm mainphase ring current: implications for skewed electric fields in the inner magnetosphere. Geophys. Res. Lett. 29, 15-1-15-3. doi: 10.1029/2002GL015160

Elfritz, J., Keesee, A., Buzulukova, N., Fok, M.-C., and Scime, E. E., (2014). First results using TWINS-derived ion temperature boundary conditions in CRCMJ. Geophys. Res. 119, 3345-3361. doi: 10.1002/2013JA019555

Fok, M.-C., Buzulukova, N. Y., Chen, S.-H., Glocer, A., Nagai, T., Valek, P., et al. (2014). The comprehensive inner magnetosphere-ionosphere model. J. Geophys. Res. 119, 7522-7540. doi: 10.1002/2014JA020239

Fok, M.-C. Buzulukova, N., Chen, S.-H., Valek, P. W., Goldstein, J., and McComas, D. J. (2010). Simulation and TWINS observations of the 22 July 2009 storm. J. Geophys. Res. 115, 1-10. doi: 10.1029/2010JA0 15443

Freeman, R. L., and Jones, E. M. (1974). Atomic Collision Processes in Plasma Physics Experiments. Culham Laboratory Report CLM-R-137.

Fuselier, S. A., Mende, S. B., Geller, S. P., Miller, M., Hoffman, R. A., Wygant, J. R., et al. (1994). Dynamics of the CRRES barium releases in the magnetosphere. J. Geophys. Res. 99, 17379-17389. doi: 10.1029/94JA01099

Goldstein, J., Angelopoulos, V., De Pascuale, S., Funsten, H. O., Kurth, W. S., LLera, K., et al. (2017). Cross-scale observations of the 2015 St. Patrick's day storm: THEMIS, van allen probes, and TWINS. J. Geophys. Res. 122, 368-392. doi: 10.1002/2016JA023173

Gruntman, M. (1997). Energetic neutral atom imaging of space plasmas. Rev. Sci. Instrum. 68, 3617.

Hughes, W. J. (1995). "The magnetopause, magnetotail, and magnetic reconnection," in Introduction to Space Physics, eds M. G. Kivelson and C. T. Russell (New York, NY: Cambridge University Press), 227-287.

Hutchinson, I. H. (1987). Principles of Plasma Diagnostics. Cambridge: Cambridge University Press.

Ilie, R., Skoug, R. M., Funsten, H. O., Liemohn, M. W., Bailey, J. J., and Gruntman, M. (2013a). The impact of geocoronal density on ring current development. J. Atmosph. Solar Terrestr. Phys. 99, 92-103. doi: 10.1016/j.jastp.2012.03.010

Ilie, R., Skoug, R. M., Valek, P., Funsten, H. O., and Glocer, A. (2013b). Global view of inner magnetosphere composition during storm time. J. Geophys. Res. 118, 7074-7084. doi: 10.1002/2012JA018468

Keesee, A., Scime, E., and Moldwin, M. (2008). Remote measurements of ion temperatures in the terrestrial magnetotail. J. Geophys. Res. 113:A00A03. doi: 10.1029/2008JA013130

Keesee, A. M., Chen, M., Scime, E. E., and Lui, A. T. Y. (2014). Regions of ion energization observed during the Galaxy-15 substorm with TWINS. J. Geophys. Res. 119, 8274-8287. doi: 10.1002/2014JA020466.

Keesee, A. M., Elfritz, J., McComas, D. J., and Scime, E. E. (2012). Inner magnetosphere convection and possible reconnection outflow of hot ions imaged during a HSS-driven storm. J. Geophys. Res. 117:A00L06. doi: 10.1029/2011JA017319

Keesee, A. M., and Scime, E. E. (2015). Database of ion temperature maps during geomagnetic storms. Earth Space Sci. 2, 39-46. doi: 10.1002/2014EA000061

McComas, D. J., Allegrini, F., Bochsler, M., Bzowski, E. R., Christian, G. B., Crew, R., DeMajistre, H., et al. (2009b). Global observations of the interstellar interaction from the Interstellar Boundary Explorer (IBEX). Science 326, 959-962. doi: 10.1126/science.1180906

McComas, D. J., Allegrini, J., Baldonado, B., Blake, P. C., Brandt, J., Burch, J., et al. (2009a). The Two Wide-angle Imaging Neutral-atom Spectrometers (TWINS) NASA mission-of-opportunity. Space Sci. Rev. 142, 157-231. doi: $10.1007 /$ s11214-008-9467-4

McComas, D. J., Funsten, H. O., Moore, K. R., Scime, E. E., and Thomsen, M. F. (1993). Detection of cold gas releases in space via low energy neutral atom imaging. Proc. SPIE 1952, 192-202.

Meier, R. R., McLaughlin, B. M., Warren, H. P., and Bishop, J. (2007). Atomic oxygen photoionization rates computed with high resolution cross sections and solar fluxes. Geophys. Res. Lett. 34:L01104. doi: 10.1029/2006GL02 8484_4

Mitchell, D. G., Jaskulek, S. E., Schlemm, C. E., Keath, E. P., Thompson, R. E., Thompson, B. E., et al. (2000). High energy neutral atom (HENA) imager for the IMAGE mission. Space Sci. Rev. 91, 67-112. doi: 10.1023/A:1005207308094

Moore, T. E., Chornay, D. J., Collier, M. R., Herrero, F. A., Johnson, J., Johnson, M. A., et al. (2000). The low energy neutral atom imager for IMAGE. Space Sci. Rev. 91, 155-195. doi: 10.1007/978-94-011-4233-5_6

Ogawa, H. S., Wu, R. C. Y., Gangopadhyay, P., and Judge, D. L. (1995). Solar photoionization as a loss mechanism of neutral interstellar hydrogen in interplanetary space. J. Geophys. Res. 100, 3455-3462.

Ostgaard, N., Mende, S. B., Frey, H. U., and Gladstone, G. R. (2003). Neutral hydrogen density profiles derived from geocoronal imaging. J. Geophys. Res. 108:1300. doi: 10.1029/2002JA 009749

Perez, J. D., Fok, M.-C., and Moore, T. E. (2000). Deconvolution of energetic neutral atom images of the Earth's magnetosphere. Space Sci. Rev. 91, 421-436. doi: 10.1023/A:1005277307611

Perez, J. D., Goldstein, J., McComas, D. J., Valek, P., Fok, M.-C., and Hwang, K.J. (2016). Global images of trapped ring current ions during main phase of 17 March 2015 geomagnetic storm as observed by TWINS. J. Geophys. Res. 121, 6509-6525. doi: 10.1002/2016JA022375

Perez, J. D., Kozlowski, G., C:son-Brandt, P., Mitchell, D. G., Jahn, J. M., Pollock, C. J., et al. (2001). Initial ion equatorial pitch angle distributions from medium and high energy neutral atom images obtained by IMAGE. Geophys. Res. Lett. 28, 1155-1158. doi: 10.1029/2000GL012636

Pollock, C. J., Asamura, K., Baldonado, J., Balkey, M. M., Barker, P., Burch, J. L., et al. (2000). Medium Energy Neutral Atom (MENA) imager for the IMAGE mission. Space Sci. Rev. 91, 113-154. doi: 10.1023/A:1005259324933

Pollock, C. J., Asamura, K., Balkey, M. M., Burch, J. L., Funsten, H. O., Grande, M., et al. (2001). First medium energy neutral atom (MENA) images of earth's magnetosphere during substorm and storm-time. Geophys. Res. Lett. 28, 1147-1150. doi: 10.1029/2000GL012641

Rairden, R. L., Frank, L. A., and Craven, J. D. (1986). Geocoronal imaging with dynamics explorer. J. Geophys. Res. 91:13613.

Roelof, E. (1987). Energetic neutral atom image of a storm-time ring current. Geophys. Res. Lett. 14, 652-655.

Roelof, E. C., Mitchell, D., and Williams, D. (1985). Energetic neutral atoms $(\mathrm{E} \sim 50 \mathrm{keV})$ from the ring current: IMP $7 / 8$ and ISEE 1. J. Geophys. Res. 90:10991-11008. doi: 10.1029/JA090iA11p10991

Scime, E. E., and Hokin, S. (1992). Design and calibration of a fast time resolution charge exchange analyzer. Rev. Sci. Instr. 63:4527. doi: 10.1063/1.1143710

Scime, E. E., Pollock, C., Jahn, J.-M., Kline, J., and Keesee, A. (2002). Ion heating in the terrestrial magnetosphere during substorms and storm-time: MENA observations. Geophys. Res. Lett. 29:1438. doi: 10.1029/2001GL013994

Scime, E. E., and Zaniewski, A. (2004). Charge exchange imaging of space plasmas. Rev. Sci. Instrum. 75, 3526-3530. doi: 10.1063/1.1779618

Shue, J.-H., Chao, J., Fu, H., Russell, C., Song, P., Khurana, K., et al. (1997). A new functional form to study the solar wind control of the magnetopause size and shape. J. Geophys. Res. 102, 9497-9511. doi: 10.1029/97JA00196

Conflict of Interest Statement: The authors declare that the research was conducted in the absence of any commercial or financial relationships that could be construed as a potential conflict of interest.

Copyright (c) 2019 Scime and Keesee. This is an open-access article distributed under the terms of the Creative Commons Attribution License (CC BY). The use, distribution or reproduction in other forums is permitted, provided the original author(s) and the copyright owner(s) are credited and that the original publication in this journal is cited, in accordance with accepted academic practice. No use, distribution or reproduction is permitted which does not comply with these terms. 